\title{
Endoscopic management of recurrent pyogenic cholangitis
}

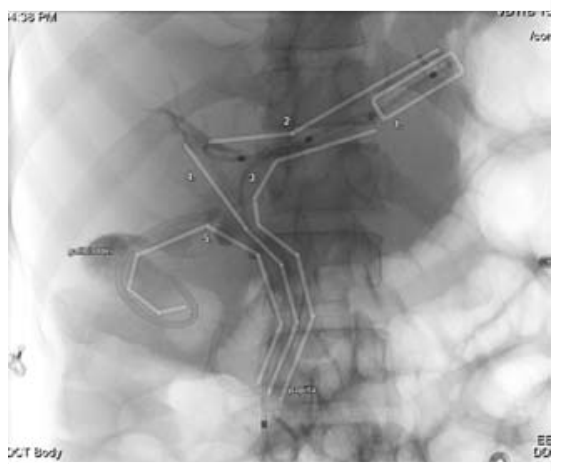

- Fig. 1 Fluoroscopic image after placement of transcystic and right intrahepatic duct (RIHD) stents showing: the hepaticogastrostomy left intrahepatic duct (LIHD) fully covered self-expandable metal stent (FCSEMS; labeled 1); the hepaticogastrostomy LIHD plastic stent (labeled 2); the transpapillary rendezvous plastic sent (labeled 3); the transpapillary RIHD plastic stent (labeled 4); the transcystic plastic stent (labeled 5).

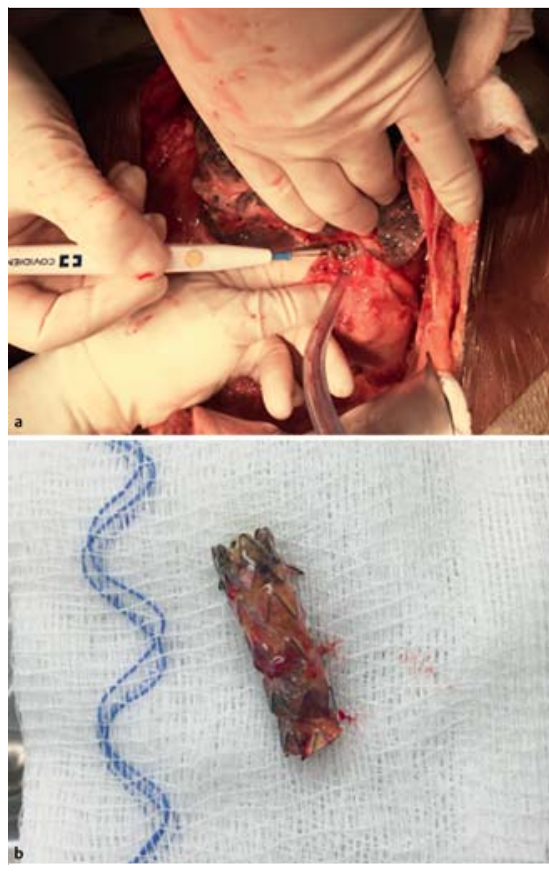

- Fig. 2 The fully covered self-expandable metal stent (FCSEMS) is seen: a at the interface between the left lobe of the liver and the adjoining stomach wall during the liver transplant procedure; b following its removal.
Recurrent pyogenic cholangitis (RPC) can be challenging to manage. The case presented illustrates all the endoscopic techniques available to us to manage this difficult entity.

A 54-year-old man with RPC presented with recurrent abdominal pain, fever, and jaundice. Magnetic resonance cholangiopancreatography (MRCP) showed an extensive stone burden. A conventional endoscopic retrograde cholangiopancreatography (ERCP) confirmed the magnetic resonance imaging (MRI) findings, but was unable to clear the extensive stone burden. Endoscopic ultrasound (EUS)-guided hepaticogastrostomy placement was performed to allow drainage of the left biliary system. After the tract had been allowed to mature, a repeat transgastric ERCP with cholangioscopic assistance was performed. Fluoroscopic and cholangioscopic visualization showed inflammation in the bile duct, but there were no stones visualized. A fully covered self-expandable metal stent (FCSEMS; $10 \mathrm{~mm} \times 6 \mathrm{~cm}$ ) with an anchoring double-pigtail stent $(7 \mathrm{Fr}, 10 \mathrm{~cm})$ were deployed, and the patient was discharged home.

After 3 months, the patient returned for stent revision. The fistulous tract was cannulated with a sphincterotome and a

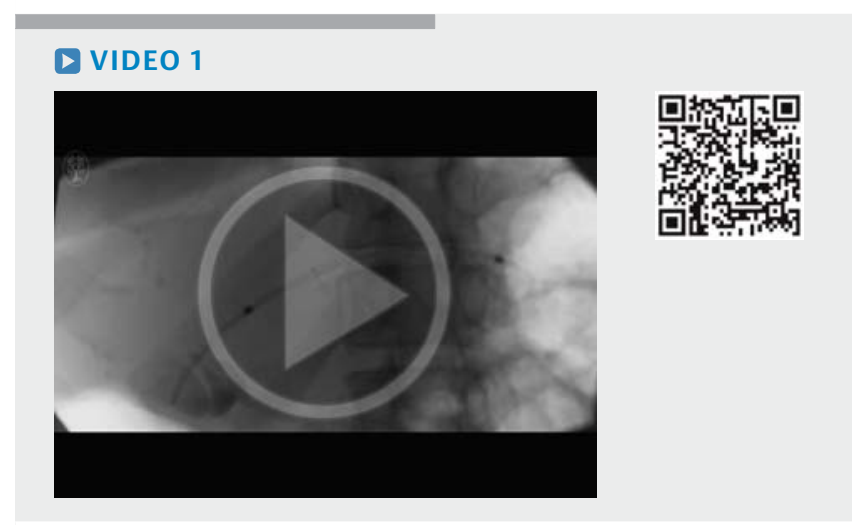

- Video 1: The different steps that were used to drain the biliary tree with a combination of transhepatic, transpapillary, and transcystic drainage. hydrophilic guidewire. The wire was advanced across the ampulla into the duodenum with a swing-tip catheter. The duodenoscope was removed over the wire to perform a rendezvous procedure. The hepaticogastrostomy stent was revised, and the patient was again discharged home. He re-presented a few months later with cholecystitis and underwent a successful ERCP with placement of a transcystic biliary drain (10 Fr, $15 \mathrm{~cm}$ ) ( Fig. 1; Video 1). His condition then improved with antibiotics, and he was again discharged home. He went on to undergo liver transplantation ( $\triangleright$ Fig.2), and was recovering at home at the time of writing.

RPC is a disease characterized by intrabiliary pigment stone formation that results in stricturing of the biliary tree and biliary obstruction with recurrent bouts of cholangitis [1]. Its etiology is thought to arise from bile stasis, transient portal bacteremia, and abnormal phospholipid metabolism. Stone clearance is the mainstay of therapy, and this can be accomplished invasively either through surgical resection, surgical T-tube placement, or non-invasively through ERCP [2]. Previous reports have indicated complete clearance of stones in $66 \%$ of cases [3]. 
This case demonstrates the successful management of a patient with RPC that involved repeated ERCPs with stent revisions using several of the available techniques. We were able to continue to decompress the biliary tree until a successful liver transplant could be performed.

Endoscopy_UCTN_Code_CCL_1AB_2AH

\section{Competing interests}

Michel Kahaleh has received grant support from Boston Scientific, Fujinon, EMcison, Xlumena Inc., W.L. Gore, MaunaKea, Apollo Endosurgery, Cook Endoscopy, ASPIRE Bariatrics, GI Dynamics, NinePoint Medical, Merit Medical, Olympus and MI Tech. He is a consultant for Boston Scientific, Xlumena Inc., Concordia Laboratories Inc, ABBvie, and MaunaKea Tech.

All other authors have no conflicts of interests to report.
The Authors

Aleksey Novikov, Nikhil Kumta, Kunal Karia, Porfirio Reinoso, Benjamin Samstein,

\section{Michel Kahaleh}

Division of Gastroenterology and Hepatology, Weil Cornell Medical College, Cornell University New York, New York, USA

\section{Corresponding author}

\section{Michel Kahaleh, MD}

Division of Gastroenterology and Hepatology, Weill Cornell Medical College, New York, NY 10021, USA,

Fax: +1-646-962-0110

mkahaleh@gmail.com

\section{References}

[1] Guyen T, Powell A, Daugherty T et al. Recurrent pyogenic cholangitis. Dig Dis Sci 2010; 55: $8-10$

[2] Cheng YF, Lee TY, Sheen-Chen SM et al. Treatment of complicated hepatolithiasis with intrahepatic biliary stricture by ductal dilatation and stenting: Long-term results. World J Surg 2000; 24: $712-716$

[3] Okugawa T, Tsuyuguchi T, Sudhamshu K et al. Peroral cholangioscopic treatment of hepatolithiasis: Long-term results. Gastrointest Endosc 2002; 56: 366 - 371

\section{Bibliography}

DOI http://dx.doi.org/10.1055/s-0042-118229

Endoscopy 2017; 49: E5-E6

(c) Georg Thieme Verlag KG

Stuttgart · New York

ISSN 0013-726X 